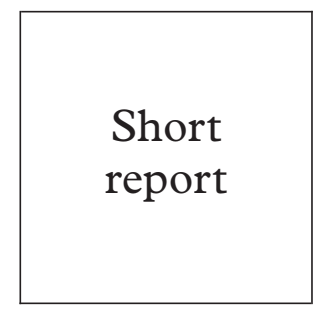

Department of Genitourinary

Medicine, St Thomas's Hospital, London A DeRuiter

E M Jungmann

Department of Sexually Transmitted Diseases, Royal Free \& University College Medical School,

London

D Mercey

Department of

Genitourinary

Medicine, King's

College Hospital,

London

S Edwards

Department of

Paediatrics, St

George's Hospital,

London

S Donoghue

Department of

Genitourinary

Medicine, Chelsea \&

Westminster Hospital,

London

$\mathrm{T}$ Booth

Department of

Paediatics, Imperial

College, London

D Mohan

H Lyall

Department of

Genitourinary

Medicine, Imperial

College, London

G P Taylor

Correspondence to:

Dr Graham P Taylor,

Department of

Genitourinary Medicine and

Communicable Diseases,

Faculty of Medicine,

Imperial College, Norfolk

Place, London W2 1PG, UK

g.p.taylor@ic.ac.uk

Accepted for publication

17 August 2001

\title{
Is first trimester exposure to the combination of antiretroviral therapy and folate antagonists a risk factor for congenital abnormalities?
}

Eva M Jungmann, Danielle Mercey, Annemiek DeRuiter, Simon Edwards, Sheila Donoghue, Tamsin Booth, Depeeka Mohan, Hermione Lyall, Graham P Taylor

Objectives: To assess use of antiretroviral therapy (ART) by HIV positive pregnant women in London since 1994 and the risk of congenital abnormalities associated with multidrug exposure during the first trimester of pregnancy.

Methods: Retrospective multicentre study of medical, obstetric, and paediatric notes of all mother-infant pairs, where the mother was known to be HIV infected before delivery, using a standardised proforma.

Results: In this study of 195 mother-infant pairs, use of ART during any stage of pregnancy increased from $33.3 \%$ in 1994 to $92.5 \%$ in $1999(\mathrm{p}=0.01$, trend). First trimester exposure increased from $0 \%$ in 1994 to $27.5 \%$ in 1999 ( $\mathrm{p}=0.00045$, trend). Congenital malformations were observed in nine infants $(4.6 \%)$. Compared with infants not exposed to ART or folate antagonists during the first trimester $(\mathrm{n}=148)$, exposure to both ART and folate antagonists during the first trimester $(n=13)$ was associated with an increased risk of congenital abnormalities ( $4 \%$ v $23.1 \%$; OR $7.10,95 \%$ CI $1.5,34.2$ ). No malformations were observed in the 34 children exposed to either ART or folate antagonists alone during the first trimester.

Conclusion: An increasing number of HIV infected women conceived while on ART. Although there is no evidence of teratogenicity caused by ART if given alone during the first trimester, exposure to the combination of ART and folate antagonists was associated with a significantly higher risk of congenital abnormalities in this cohort.

(Sex Transm Inf 2001;77:441-443)

Keywords: antiretroviral therapy; folate antagonists; congenital abnormalities

\section{Introduction}

The epidemiology of the human immunodeficiency virus (HIV)-1 in the United Kingdom is changing and in 1999 heterosexually acquired newly diagnosed HIV infections exceeded those in men who have sex with men, for the first time. ${ }^{1}$ Thus, an increasing number of women have to balance reproductive and therapeutic (antiretroviral) choices. While vertical transmission of HIV-1 can be reduced to less than $2 \%,{ }^{23}$ there are few published data on the safety of antiretroviral therapy (ART) to

\section{Key messages}

- First trimester exposure to currently licensed antiretroviral therapy has not so far been associated with human teratogenesis

- Folate antagonists, which are associated with congenital malformations, are commonly prescribed to women with advanced HIV infection

- The risk of congenital malformations in children born to HIV infected mothers may be increased by multiple drug exposure.

- All women taking antiretroviral therapy during pregnancy should be reported to the antiretroviral pregnancy registry prospectively human embryos/fetuses. A large North American study found the risk of congenital malformations with zidovudine exposure increased with later rather than first trimester exposure but was confounded by high rates of injecting drug, alcohol, and tobacco use among the mothers. ${ }^{4}$ Worldwide surveillance through the International Antiretroviral Pregnancy Registry $^{5}$ has so far revealed no excess risk of congenital abnormalities in infants exposed to ART during pregnancy. However, the rates of congenital malformations in the prospective arm of the registry are lower with first trimester than with second/third trimester exposure and lower than that reported from the Metropolitan Atlanta Congenital Defects Program. ${ }^{6}$ Furthermore many HIV infected women take other potentially teratogenic therapies, such as the folate antagonists prescribed for Pneumocystis carinii pneumonia (PCP) prophylaxis, in addition to ART, and this information is not currently collected.

In this study, we evaluated the risk of congenital abnormalities associated with multidrug exposure during the first trimester of pregnancy in infants of HIV infected mothers.

\section{Methods}

We retrospectively collected maternal HIV, obstetric, and paediatric data on a cohort of mother-infant pairs where the mother was known to be HIV infected before delivery and delivered at one of six inner London hospitals 
Table 1 Antepartum and first trimester drug exposure ( $n=195)$

\begin{tabular}{lll}
\hline Drug exposure & 1st trimester exposure (\%) & Any antepartum exposure (\%) \\
\hline ART & $28(14.4 \%)$ & $167(85.6 \%)$ \\
Zidovudine monotherapy & $2(7.1 \%)$ & $89(45.6 \%)$ \\
Dual NRTI & $12(42.9 \%)$ & $25(12.8 \%)$ \\
Dual NRTI + NNRTI & $3(10.7 \%)$ & $26(13.3 \%)$ \\
Dual NRTI + PI & $9(32.2 \%)$ & $22(11.3 \%)$ \\
All three ART classes & $2(7.1 \%)$ & $5(2.6 \%)$ \\
Other medication & $44(22.6 \%) \dagger$ & $84(43.1 \%)$ \\
Folate antagonistsł & $32(16.4 \%)$ & $57(29.2 \%)$ \\
ART and folate antagonists & $13(6.7 \%)$ & $51(26.2 \%)$ \\
\hline
\end{tabular}

ART $=$ antiretroviral therapy, NRTI $=$ nucleoside analogue reverse transcriptase inhibitor, NNRTI = non-nucleoside analogue reverse transcriptase inhibitor, PI = protease inhibitor. $\star$ Percentages in italics refer to exposure subgroup.

†Includes folate antagonists (32), aciclovir (2), benzodiazepine (4), methadone (4), amitryptiline, chloroquine, prednisolone, dapsone (2), rifamycins (4), isoniazid (2), pyrazinamide, ethambutol, erythromycin, ferrous sulphate (2), thyroxine, cephalosporin, clarithromycin, metronidazole, nitrofurantoin, roaccutane, ranitidine, atenolol, enalapril.

‡Includes co-trimoxasole (29), pyrimethamine (2), carbamezepine (1).

between 1 May 1994 and 30 June 1999. All such women, including those who had a medically indicated late termination of pregnancy or in utero death/miscarriage after 20 weeks of gestation, were included.

Data were extracted from case notes using a pre-agreed proforma and included maternal age, ethnicity, CD4 count (lowest) during first trimester (0-12 gestational weeks), antiretroviral and other drug therapy per trimester of pregnancy. All structural defects present at birth were recorded as congenital malformations. Data were entered into Microsoft Access software and analysed with STATA 5 statistical software.

Frequency distributions were derived for sociodemographic data, potential risk factors for birth defects, and uptake of therapy during pregnancy. First trimester exposure was divided into three categories to reflect the number of drug classes: no exposure (baseline), exposure to folate antagonists only or ART only, and exposure to folate antagonists and ART. CD4 counts were categorised into two groups to reflect the severity of immunosuppression ( $\geqslant 200 \times 10^{6} / 1$ and $\left.<200 \times 10^{6} / 1\right)$. The primary outcome was defined as the presence of congenital abnormalities at birth. The association of categorical variables with the outcome was assessed using $\chi^{2}$ significance testing and of maternal age using $t$ test. In univariate analysis, the Mantel-Haenszel method was used to calculate crude odds ratios (OR) for each first trimester exposure category compared with the baseline category. Multivariate analysis was not performed because of the small number of outcomes.

\section{Results}

Of the 211 eligible mother-infant pairs, medical records were missing or incomplete for 16, leaving 195 mother-infant pairs (92.4\%) available for analysis. Mean age of the mothers at delivery was 29 years (SD 4.7), 137 (70.3\%) were multiparous, and the median $\mathrm{CD} 4$ count during the first trimester was $255 \times 10^{6} / 1$ (IQR $150-400, \mathrm{n}=177)$. The majority $(156 / 195$, $80 \%$ ) were black African and 14.4\% (28/195) were white.

ART during pregnancy increased from $33.3 \%(4 / 12)$ in 1994 to $84 \%$ (31/37) in 1996 and remained high at $92.5 \%(37 / 40)$ during the first 6 months of $1999(\mathrm{p}=0.01$, trend). First trimester exposure to ART increased from $0 \%$ $(0 / 12)$ in 1994 to $20.4 \%$ (10/49) in 1998 and to $27.5 \%(11 / 40)$ in the first 6 months of 1999 $(p=0.0004$, trend). Eighty four $(43.1 \%)$ infants were exposed to other medication during pregnancy; this includes $44(22.6 \%)$ infants exposed during the first trimester. Table 1 shows details of antepartum drug exposure.

In the whole cohort, nine children $(4.6 \%$, $95 \%$ CI 1.7, 7.5) with congenital abnormalities were reported (Table 2). Compared with the 148 infants not exposed to ART or folate antagonists during the first trimester, first trimester exposure to both therapies $(n=13)$ was associated with a sevenfold increased risk of congenital abnormalities $(6 / 148(4 \%) v 3 / 13$ (23.1\%); OR 7.10, 95\% CI 1.5, 34.2). No congenital abnormalities were observed in the 34 infants exposed to either ART alone or folate antagonists alone during the first trimester.

The development of congenital abnormalities was not associated with a maternal CD4 count below $200 \times 10^{6} / 1(\mathrm{p}=0.27)$, maternal age $(p=0.11)$, or ethnicity $(p=0.81)$. However, a higher proportion of mothers taking ART and folate antagonists $(61.5 \%)$ or folate antagonists alone $(59.4 \%)$ during the first trimester had CD 4 counts $<200 \times 10^{6} / 1$ compared with those mothers not on therapy $(22.6 \%, \mathrm{p}<0.001)$.

\section{Discussion}

As demonstrated here an increasing number of conceptions are being exposed to antiretroviral

Table 2 Description of maternal CD4 count, antiretroviral and folate antagonist (co-trimoxazole, pyrimethamine) exposure and reported congenital abnormalities in their infants

\begin{tabular}{|c|c|c|c|c|c|}
\hline Child & $\begin{array}{l}\text { Maternal CD4 } \\
\text { count }\left(\times 10^{6} / l\right)^{*}\end{array}$ & $A R T$ & $\begin{array}{l}\text { Trimester of } \\
\text { exposure }\end{array}$ & $\begin{array}{l}\text { 1st trimester } \\
\text { folate } \\
\text { antagonist }\end{array}$ & Congenital abnormality \\
\hline 1 & 831 & $\mathrm{AZT}$ & 3 & No & Hydrocele \\
\hline 2 & 250 & $\mathrm{AZT}$ & $2-3$ & No & Polydactyly \\
\hline 3 & N/A & $\mathrm{AZT}$ & 3 & No & Hydronephrosis \\
\hline 4 & 200 & $\mathrm{AZT}$ & 3 & No & Polydactyly, cryptorchism \\
\hline 5 & 143 & $\mathrm{AZT}$ & Intrapartum & No & Absent left testes \\
\hline 6 & 204 & AZT, ddI, nevirapine & 3 & No & Fallot's tetralogy, DiGeorge syndrome \\
\hline $7 \ddagger$ & 60 & $\mathrm{AZT}, \mathrm{ddC}$ & $1-3$ & Yes $§$ & Hemivertebrae, butterfly vertebrae \\
\hline $8 \dagger \neq$ & 29 & ddI, d4T, nevirapine & $1-2$ & Yes & Spina bifida, hydrocephalus \\
\hline 9 & 60 & $3 \mathrm{TC}, \mathrm{d} 4 \mathrm{~T}$ & $1-3$ & Yesף & Exomphalos \\
\hline
\end{tabular}

AZT $=$ zidovudine, $\mathrm{ddI}=$ didanosine, $\mathrm{ddC}=$ zalcitabine, $3 \mathrm{TC}=$ lamivudine, $\mathrm{d} 4 \mathrm{~T}=$ stavudine

^Lowest during first trimester.

$\dagger$ Medically indicated termination at $21 / 40$.

$\neq$ For details see Richardson et al. ${ }^{10}$

\$First trimster rifampicin, isoniazid, pyridoxine.

ๆFirst trimester dapsone. 
and other HIV related therapies during the first trimester of pregnancy.

The overall risk of congenital abnormalities $(4.6 \%, 95 \%$ CI $1.7,7.5)$ in this study was similar to that seen in the Antiretroviral Pregnancy Registry ${ }^{5}$ and no excess risk was attributed to ART alone. However, in our cohort, $23 \%$ of infants in the subgroup of those exposed to both folate antagonists and ART during the first trimester $(n=13)$ were born with congenital abnormalities, some requiring major surgery. Although the numbers are small, they had a sevenfold increased risk of congenital abnormalities compared with infants not exposed to any drugs during the first trimester (OR 7.10, 95\% CI 1.5, 34.2).

In women with advanced immunosuppression dihydrofolate reductase inhibitors (cotrimoxazole, pyrimethamine) are prescribed for PCP prophylaxis. A threefold increase in cardiovascular defects, oral clefts, and urinary tract defects has been reported in infants exposed to dihydrofolate reductase inhibitors (short or long term exposure) during organogenesis. ${ }^{7}$ The risk was significantly reduced by supplementation with folic acid-containing multivitamins. Periconceptual folic acid supplementation has been shown to reduce the risk of neural tube defects ${ }^{8}$ and is now recommended but a protective effect in women on long term folate antagonists has not been established.

This study has several limitations. Owing to its retrospective nature we were unable to collect reliable information on folic acid supplementation and medication prescribed by other service providers in early pregnancy. In addition, data on intravenous and illicit drug use, self medication, nutritional status, and familial predisposition were not available. However injecting drug use is anecdotally rare in black African communities in the United Kingdom and only four women $(2.0 \%)$ were taking benzodiazepines or methadone. Although the mothers of three of the four children with serious malformations had CD4 counts below 200 $\times 10^{6} / 1$, severe immunosuppression has so far not been associated with fetal abnormalities and is unlikely to be a confounder. ${ }^{9} 10$
Until more safety data are available the need for primary prophylaxis with folate antagonists should be regularly reviewed in women of childbearing age and the benefits of folic acid supplementation emphasised during preconceptual counselling. Existing surveillance programmes of (ART) exposed children should include details of all medication and timing of the exposure to allow identification of risk factors and subgroups at increased risk of congenital malformations. These findings, if confirmed, have important implications for preconceptual counselling and the therapeutic choices of women of childbearing age.

Conflict of interest: None.

We are grateful to Pat Tookey (Institute of Child Health, London) for providing data from the BPSU surveillance and to Eimear Canavan (King's College Hospital, London) for assisting in the data collection.

Contributors: ADR, DaM, HL, and GPT developed the idea of the study and the study design. EMJ coordinated the study and data collection, conducted the analysis and interpretation, set up the database together with DeM and prepared the first draft of the database together with DeM and prepared the first draft of
the paper. All authors were actively involved in data collection and participated in the writing of the article.

1 PHLS Communicable Disease Surveillance Centre. AIDS and HIV infection: monthly report. CDR Weekly 2000; 10:77-80

2 Lindegren ML, Byers RH, Thomas P, et al. Trends in perinatal transmission of HIV/AIDS in the United States. fAMA 1999;282:531-8.

3 Mofenson LM. Public Health Service Task Force recommendations for the use of antiretroviral drugs in pregnant women infected with HIV-1 for maternal health and for reducing perinat HIV-1 transmission in the United States. Perinatal HIV Guidelines Working Group, CDC, 4 May 2001.

4 Newschaffer CJ, Cocroft J, Anderson CE, et al. Prenatal zidovudine use and congenital anomalies in a Medicaid population. F Acquir Immune Defic Syndr 2000;24:249-56.

5 The Antiretroviral Pregnancy Registry (Interim Report) 1 Jan 1989-31 Jul 2000. Wilmington, NC: Registry Project Office.

6 Metropolitan Atlanta Congenital Defects Program surveillance data, 1988-1991. Teratology 1993;48:695-709.

7 Hernandez-Diaz S, Werler MM, Walker AM, et al. Folic acid antagonists during pregnancy and the risk of birth defects. N Engl f Med 2000;343:1608-14.

8 MRC Vitamin Study Research Group. Prevention of neural tube defects: results of the Medical Research Council vitamin study. Lancet 1991;338:131-7.

9 Ades AE, Newell M-L, Peckham C. Children born to women with HIV-1 infection. Natural history and risk of transmission. European Collaborative Study. Lancet 1991; 337:252-60

10 Richardson M, Osrin D, Donaghy S, et al. Spinal malformations in the fetuses of HIV infected women receiving combination antiretroviral therapy and co-trimoxazole. Eur $\mathcal{F}$ Obstet Gynecol Reprod Biol 2000;93:215-17. 